\title{
Effects on Tree Vigor, Fruit Quality, Yield and Fruit Fly Infestation of Different Time of Pruning with Genotypes in Guava (Psidium guajava L.)
}

\author{
P. H. Nikumbhe ${ }^{1 *}$, J. Uchoi ${ }^{2}$ and D. S. Mali ${ }^{3}$ \\ ${ }^{1}$ Department of Horticulture, Post Graduate Institute, MPKV, Rahuri, (MS), India \\ ${ }^{2}$ Current Institute: ICAR-National Research Centre for Grapes; Pune, (MS), India \\ ${ }^{3}$ ICAR-NBPGR, Regional Station; Shillong (Meghalaya), India \\ *Corresponding author
}

\section{A B S T R A C T}

\section{Keywords}

Fruit quality and

Yield, Hast bahar,

Psidium guajava,

Pruning time

Article Info

Accepted:

15 October 2020

Available Online:

10 November 2020
Poor quality and fruit fly infested fruit in the rainy season and failure to manipulate production periods are common problems for guava production in India. So, the research work was carried out with a management practices point of view to overcome these problems. Growth characters were significantly influenced by different genotypes. The plant spread, number of sprouted shoots was recorded maximum in cv. Sardar. Marketable yield free from fruit fly infestation were significantly higher recorded in $15^{\text {th }}$ July time of pruning and its interaction with cv. Sardar. As concerned with qualitative attributes the genotype Sardar was observed better in T.S.S. as compared to other genotypes. RHR-Guv14 genotype was found to be maximum in ascorbic acid. Sugar: acid ratio was observed higher in RHR-Guv-60. Similarly maximum shelf life of fruit was recorded by the RHRGuv-14. Very crispy pulp texture and fruit luster was observed in all genotypes except cv. Sardar. From the present investigation, it was found that $15^{\text {th }}$ July pruning time was found to be better with respect to marketable yield. Pruning time of $15^{\text {th }}$ September was found to be better in the escape from fruit fly infestation but fruiting was very less. The genotype RHR-Guv-14 may evaluate for the cultivation as hast bahar crop.

\section{Introduction}

Guava fruit is often called "poor man's apple" though the fruit is neither poor in its nutritive value and nor commercial value, contributes $3.4 \%$ of total fruit area and $3.9 \%$ of total fruit production in India during 2013-14 (Anon., 2014). Guava is one of the richest natural sources of vitamin $\mathrm{C}$ contains 2 to 5 times more vitamin $\mathrm{C}$ than oranges and 10 times more than tomato. Compared to other fruits, the whole guava is a moderately good source of calcium, a fair source of phosphorus and a good source of iron. Guava is consumed in different ways. The fruit has sweet aroma wholly edible along with the skin. Seeds yield 3 to 13 percent oil, which rich in essential fatty acid and can be used as a salad dressing (Mahor et al., 2012), usually eaten raw both green and ripe (when it becomes fragrant). It is also stewed and used in shortcakes, puddings, sauce, ice cream, butter, 
marmalade, chutney and other products and pies. The fruits produced during the rainy season are severely attacked by the seasonal insect called fruit fly. Infestation of fruit flies ranges from 20 to 46 percent with a crop loss of 16 to 40 percent, which is the matter of serious concern (Hasseb, 2007). The major objective of the present work is to study the influence of pruning and genotypes on quality, yield of fruit and incidence of the fruit fly.

\section{Materials and Methods}

Research work was carried out at the "Instructional-cum-Research Orchard" of the Department of Horticulture, MPKV, Rahuri, Dist. Ahmednagar, during the year 2013 and 2014. The soil of the experimental field was light to medium in texture with good drainage within the depth 0.2 to $0.4 \mathrm{~m}$. The annual rainfall ranges from 307 to $619 \mathrm{~mm}$ with an average of $520 \mathrm{~mm}$. Genotypes were planted with Spacing of $6 \times 6 \mathrm{~m}$ in the year of 2006 . Six years old guava plants were selected in the experiment. The treatment includes Factor A: Seven Genotypes of 7-8 years old i.e. Sardar $\left(\mathrm{S}_{1}\right)$, RHR-Guv-58 $\left(\mathrm{S}_{2}\right)$, RHR-Guv-60 $\left(\mathrm{S}_{3}\right)$, RHR-Guv-14 ( $\left.\mathrm{S}_{4}\right)$, RHR-Guv-16 $\left(\mathrm{S}_{5}\right)$, RHR-Guv-3 ( $\left.\mathrm{S}_{6}\right)$ and RHR-Guv-6 $\left(\mathrm{S}_{7}\right)$. Factor $\mathrm{B}:$ Six pruning time i.e. $15^{\text {th }}$ May $\left(\mathrm{P}_{1}\right), 15^{\text {th }}$ June $\left(\mathrm{P}_{2}\right), 15^{\text {th }}$ July $\left(\mathrm{P}_{3}\right), 15^{\text {th }}$ August $\left(\mathrm{P}_{4}\right), 15^{\text {th }}$ Sept $\left(\mathrm{P}_{5}\right)$ and Control $\left(\mathrm{P}_{0}\right)$.The experiment was laid out in the factorial randomized block design with forty-two treatments replicated two times. In the experiment, 75 percent pruning of current season growth of guava trees were pruned at monthly intervals.

\section{Results and Discussion}

\section{The height of plant}

The maximum height of plant was recorded in $\mathrm{P}_{0}$ (Control) $(2.30 \mathrm{~m})$ treatment. The height of plant was observed non-significant for genotypes and interaction among the pruning time and different genotypes (Table 1). There is an increase in height of plants after the pruning operation as compared to control one. It might be ascribed as a faster growth of newly sprouted shoots of pruned trees due to the availability of stored carbohydrates to the plant. The results of present studies are confirmed with those of Basu et al., (2007) who also reported the significant increase in guava plant height after pruning as compared to control.

\section{Plant spread}

Significantly maximum East-West $(5.89 \mathrm{~m})$ and North-South $(5.99 \mathrm{~m})$ plant spread was recorded in $\mathrm{S}_{1}$ (Sardar) genotype. East-West and North-South plant spread was observed non-significant for pruning time and for interaction among the pruning time and different genotypes. Increase in spread (EW and NS) was observed in all pruning treatments and in all genotypes as compared to control (Table 2 and 3). This might be due to a high growth rate of newly emerged shoots after the pruning which leads to increase in plant spread as compared to control. Basu et al., (2007) also reported a significant increase in guava plant spread after pruning as compared to control.

\section{Number of sprouted shoots per tree}

As regards the data on pruning time, significantly maximum shoots (89.21) were recorded in $\mathrm{P}_{0}$ (control). In case of genotype maximum shoots (84.67) were noticed in $S_{1}$ (Sardar). Regarding interaction, significantly maximum number of shoots sprouted per plant was recorded in $\mathrm{P}_{0} \mathrm{~S}_{1}$ (116.00) treatment combination in pooled results (Table 4 ). The results of the conducted experiment show that the growth of control trees was more due to continuous growth habit of guava plant and pruned trees put forth more number of shoots. 
This might be due to the translocation of metabolites and favors the more sprouting in pruned matured shoots. The results of present studies are found in line with those of Singh et al., (2001) observed a maximum number of shoots in pruned trees compared to unpruned ones in guava. Dhaliwal et al., (2014) reported that pruned trees of Kinnow produce a maximum number of shoots as compared to control one.

\section{The average weight of fruit}

With respect to pruning time, the highest average weight (238.2 g) of fruit was noticed in $\mathrm{P}_{2}\left(15^{\text {th }}\right.$ June $)$. As regards to genotypes, a highest average weight of fruit $250.9 \mathrm{~g}$ ) was observed $\mathrm{S}_{4}$ (RHR-Guv-14). Significant interaction effect of pruning time and genotypes, with the highest average weight of fruit was noted in $\mathrm{P}_{2} \mathrm{~S}_{4}(274.5 \mathrm{~g})$ treatment combination in pooled results (Table 5). Results of the experiment show that maximum average weight of fruit was recorded by $S_{4}$ (genotype) and least in $S_{1}$ Genotypes which had pruned, control trees having less weight of fruit. The production of heavier fruits by trees subjected to pruning might be ascribed to the lesser crop load per tree and more nutrient supply to the limited fruit number. Discussion in further strengthened by the fact that trees subjected to pruning 75 percent removal of current season growth) might have produced more leaves/fruit ratio as compared to the control ones thereby increasing the fruit weight. More or less, similar results were also reported by Sundarajan and Muthuswamy (1964a) and Bajpai et al., (1973) in guava as they obtained an increment in fruit weight in pruned trees than control one.

\section{Length of fruit}

As regards the data on pruning time, significantly maximum length of fruit (7.21 $\mathrm{cm})$ was recorded in $\mathrm{P}_{4}$ (15 ${ }^{\text {th }}$ Aug.) in pooled results and in case of genotype maximum length of fruit (7.28) was recorded in $\mathrm{S}_{4}$ (RHR-Guv-14)). Regarding interaction, the maximum length of fruit was recorded in $\mathrm{P}_{4} \mathrm{~S}_{4}$ $(7.55 \mathrm{~cm})$ treatment combination (Table 6). In the conducted experiment, there was not too much effect of various pruning time observed on length fruit; $\mathrm{S}_{4}$ genotypes has a maximum length of fruit and least in S1 as well as in control ones.

This might be due to the effect of pruning causes shifting of metabolites in sprouted shoots which leads to increase in vegetative and reproductive growth in plants and due to which length of fruit is increased. The results of present studies are in consonance with those of Arvindakshan (1963), Sundarajan and Muthuswamy (1964a), Lotter and Lotter (1990) and Lal et al., (2000), who also obtained increased fruit size in pruned trees as compared to control ones.

\section{The diameter of fruit}

The diameter of fruit, as regards pruning time, the significantly maximum diameter of fruit $(7.24 \mathrm{~cm})$ was recorded in $\mathrm{P}_{4}\left(15^{\text {th }}\right.$ Aug.) in pooled results and regarding genotypes, the maximum diameter of fruit (7.31) was recorded in $\mathrm{S}_{4}$ (RHR-Guv-14)). In case of interaction, the significantly maximum diameter of fruit was recorded in $\mathrm{P}_{4} \mathrm{~S}_{5}(7.70$ $\mathrm{cm})$. In the experimental results of genotypes, in that $\mathrm{S}_{4}$ treatment has maximum fruit diameter (Table 7). There was not too much effect of various time of pruning observed.

Maximum fruit diameter was recorded in the trees of pruned ones than control. This is might be due to the availability of stored food to the sprouted shoots fruit buds. The present studies results are in the line with those. Aravindakshan (1963), Sundarajan and Muthuswamy (1964a), Lotter and Lotter 
(1990) and Lal et al., (2000), who also obtained increased fruit size in pruned trees. Likewise, Fivaz and Stassen (1994) recorded an improvement in fruit size with pruning in mango.

\section{Average number of seeds per fruit}

As regards time of pruning, the significant minimum average number of seeds (268) was observed in $\mathrm{P}_{3}\left(15^{\text {th }}\right.$ July) in pooled results and the minimum average number of seeds (248.5) was observed in $\mathrm{S}_{2}$ (RHR-Guv-58) genotype (Table 8). In the results of conducted experiment observed no such effect of pruning time on a number of seeds per fruit. However, genotypes having its independent characteristics regarding a number of seeds in fruits.

Table.1 Effect of pruning time and genotypes on height of plant (m)

\begin{tabular}{|c|c|c|c|c|c|c|c|c|}
\hline Treats. & & & & uava & otype & & & \\
\hline Pruning Time & S1 & S2 & S3 & S4 & S5 & S6 & S7 & Mean \\
\hline P1 & 2.14 & 1.85 & 1.53 & 1.55 & 1.77 & 1.83 & 1.60 & 1.75 \\
\hline P2 & 1.76 & 1.77 & 1.50 & 1.65 & 1.74 & 1.65 & 1.55 & 1.66 \\
\hline P3 & 2.23 & 1.70 & 1.85 & 1.75 & 1.82 & 1.59 & 1.50 & 1.78 \\
\hline P4 & 1.58 & 1.54 & 1.59 & 1.55 & 1.73 & 1.48 & 1.83 & 1.61 \\
\hline P5 & 1.80 & 1.74 & 1.79 & 1.38 & 1.24 & 1.62 & 1.60 & 1.59 \\
\hline P0 (Control) & 2.11 & 2.37 & 2.75 & 1.98 & 2.14 & 2.35 & 2.40 & 2.30 \\
\hline Mean & 1.93 & 1.83 & 1.83 & 1.64 & 1.74 & 1.75 & 1.75 & 1.78 \\
\hline Year 2013 \& 2014 & Prun & Time & & a genc & & & action & $\times S)$ \\
\hline & & & & Pooles & & & Poole & \\
\hline $\mathrm{SE}(\mathrm{m}) \pm$ & & & & 0.118 & & & 0.29 & \\
\hline CD 5\% & & & & NS & & & NS & \\
\hline
\end{tabular}

Table.2 Effect of pruning time and genotypes on plant spread (m)

\begin{tabular}{|c|c|c|c|c|c|c|c|c|}
\hline \multirow{3}{*}{$\begin{array}{l}\text { Treats. } \\
\text { Pruning Time }\end{array}$} & \multicolumn{8}{|c|}{ East-west plant spread (cm) } \\
\hline & \multicolumn{8}{|c|}{ Guava genotypes } \\
\hline & S1 & S2 & $\mathbf{S 3}$ & S4 & S5 & S6 & S7 & Mean \\
\hline P1 & 6.40 & 4.05 & 3.95 & 3.90 & 3.61 & 2.80 & 3.60 & 4.04 \\
\hline P2 & 4.96 & 3.40 & 3.33 & 3.75 & 5.27 & 3.56 & 3.15 & 3.91 \\
\hline P3 & 6.21 & 3.00 & 4.15 & 3.69 & 3.72 & 3.36 & 3.50 & 3.95 \\
\hline P4 & 5.76 & 3.21 & 3.61 & 3.66 & 4.60 & 3.74 & 3.21 & 3.97 \\
\hline P5 & 6.01 & 3.52 & 3.76 & 3.80 & 4.75 & 3.63 & 3.00 & 4.07 \\
\hline P0 (Control) & 6.02 & 3.40 & 3.42 & 4.15 & 4.60 & 3.26 & 4.13 & 4.14 \\
\hline Mean & 5.89 & 3.43 & 3.70 & 3.82 & 4.42 & 3.39 & 3.43 & 4.01 \\
\hline \multirow[t]{2}{*}{ Year $2013 \& 2014$} & \multicolumn{2}{|c|}{ Pruning Time } & \multicolumn{3}{|c|}{ Guava genotypes } & \multicolumn{3}{|c|}{ Interaction $(\mathrm{P} \times \mathrm{S})$} \\
\hline & \multicolumn{2}{|c|}{ Pooled } & \multicolumn{3}{|c|}{ Pooled } & \multicolumn{3}{|c|}{ Pooled } \\
\hline $\mathrm{SE}(\mathbf{m}) \pm$ & \multicolumn{2}{|c|}{0.208} & \multicolumn{3}{|c|}{0.225} & \multicolumn{3}{|c|}{0.552} \\
\hline CD 5\% & \multicolumn{2}{|c|}{ NS } & \multicolumn{3}{|c|}{0.624} & \multicolumn{3}{|c|}{ NS } \\
\hline
\end{tabular}


Table.3 Effect of pruning time and genotypes on plant spread (m)

\begin{tabular}{|c|c|c|c|c|c|c|c|c|}
\hline \multirow{3}{*}{\begin{tabular}{|l|} 
Treats. \\
Pruning Time \\
\end{tabular}} & \multicolumn{8}{|c|}{ North-South plant spread (cm) } \\
\hline & \multicolumn{8}{|c|}{ Guava genotypes } \\
\hline & S1 & $\mathbf{S 2}$ & S3 & S4 & S5 & S6 & S7 & Mean \\
\hline P1 & 6.15 & 4.35 & 3.40 & 3.60 & 3.51 & 3.46 & 3.10 & 3.94 \\
\hline $\mathbf{P 2}$ & 5.80 & 2.80 & 3.61 & 4.15 & 4.16 & 3.75 & 3.31 & 3.94 \\
\hline P3 & 6.41 & 3.71 & 4.36 & 3.77 & 4.28 & 3.43 & 3.15 & 4.16 \\
\hline P4 & 6.06 & 4.51 & 3.21 & 3.00 & 3.46 & 4.06 & 3.85 & 4.02 \\
\hline P5 & 5.71 & 3.50 & 4.62 & 3.75 & 3.60 & 4.10 & 3.16 & 4.06 \\
\hline P0 (Control) & 5.79 & 3.15 & 3.06 & 3.82 & 3.70 & 4.28 & 3.74 & 3.93 \\
\hline Mean & 5.99 & 3.67 & 3.71 & 3.68 & 3.78 & 3.84 & 3.38 & 4.01 \\
\hline \multirow{2}{*}{ Year 2013 \& 2014} & \multicolumn{2}{|c|}{ Pruning Time } & \multicolumn{3}{|c|}{ Guava genotypes } & \multicolumn{3}{|c|}{ Interaction $(\mathrm{P} \times \mathrm{S})$} \\
\hline & \multicolumn{2}{|c|}{ Pooled } & \multicolumn{3}{|c|}{ Pooled } & \multicolumn{3}{|c|}{ Pooled } \\
\hline $\mathrm{SE}(\mathrm{m}) \pm$ & \multicolumn{2}{|c|}{0.187} & \multicolumn{3}{|c|}{0.202} & \multicolumn{3}{|c|}{0.494} \\
\hline CD $5 \%$ & \multicolumn{2}{|c|}{ NS } & \multicolumn{3}{|c|}{0.559} & \multicolumn{3}{|c|}{ NS } \\
\hline
\end{tabular}

Table.4 Effect of pruning time and genotypes on number of sprouted shoots per tree

\begin{tabular}{|c|c|c|c|c|c|c|c|c|}
\hline Treats. & & & & Guava & otypes & & & \\
\hline Pruning Time & S1 & S2 & S3 & S4 & S5 & S6 & S7 & Mean \\
\hline P1 & 85.00 & 45.00 & 22.00 & 42.00 & 35.00 & 32.00 & 36.00 & 42.43 \\
\hline $\mathbf{P 2}$ & 73.00 & 28.00 & 27.00 & 29.00 & 26.00 & 32.00 & 33.00 & 35.43 \\
\hline P3 & 76.00 & 35.00 & 25.00 & 26.00 & 32.00 & 31.00 & 30.00 & 36.43 \\
\hline P4 & 91.00 & 37.00 & 32.00 & 32.00 & 36.00 & 22.00 & 25.00 & 39.29 \\
\hline P5 & 67.00 & 22.00 & 23.00 & 31.00 & 33.00 & 36.00 & 22.00 & 33.43 \\
\hline P0 (Control) & 116.00 & 97.50 & 75.50 & 75.50 & 91.00 & 75.50 & 93.50 & 89.21 \\
\hline Mean & 84.67 & 44.08 & 34.08 & 39.25 & 42.17 & 38.08 & 39.92 & 46.04 \\
\hline Year $2013 \& 2014$ & Pruni & Time & & a geno & & & action ( & \\
\hline & & & & Pooled & & & Pooled & \\
\hline $\mathrm{SE}(\mathrm{m}) \pm$ & & & & 0.56 & & & 1.38 & \\
\hline CD 5\% & & & & 1.56 & & & 3.84 & \\
\hline
\end{tabular}

Table.5 Effect of pruning time and genotypes on average weight of fruit (g)

\begin{tabular}{|c|c|c|c|c|c|c|c|c|}
\hline \multirow{2}{*}{$\begin{array}{l}\text { Treats. } \\
\text { Pruning Time }\end{array}$} & \multicolumn{8}{|c|}{ Guava genotypes } \\
\hline & S1 & S2 & S3 & S4 & S5 & S6 & S7 & Mean \\
\hline P1 & 165.0 & 227.0 & 246.0 & 247.5 & 227.5 & 250.0 & 205.0 & 224.0 \\
\hline $\mathbf{P 2}$ & 167.0 & 249.0 & 258.5 & 274.5 & 226.5 & 245.5 & 247.0 & 238.2 \\
\hline P3 & 179.0 & 240.5 & 225.5 & 259.5 & 224. & 246.5 & 230.0 & 229.3 \\
\hline P4 & 165.0 & 235.0 & 251.5 & 231.5 & 250.5 & 232.0 & 242.0 & 229.6 \\
\hline P5 & 163.0 & 268.5 & 231.5 & 240.5 & 234.0 & 240.0 & 214.5 & 227.4 \\
\hline P0(Control) & 161.0 & 222.0 & 242.0 & 252.0 & 241.0 & 249.5 & 233.0 & 228.6 \\
\hline Mean & 166.6 & 240.3 & 242.5 & 250.9 & 234.0 & 243.9 & 228.5 & 229.5 \\
\hline \multirow[t]{2}{*}{ Year $2013 \& 2014$} & \multicolumn{2}{|c|}{ Pruning Time } & \multicolumn{3}{|c|}{ Guava genotypes } & \multicolumn{3}{|c|}{ Interaction $(\mathrm{P} \times \mathrm{S})$} \\
\hline & \multicolumn{2}{|c|}{ Pooled } & \multicolumn{3}{|c|}{ Pooled } & \multicolumn{3}{|c|}{ Pooled } \\
\hline $\mathrm{SE}(\mathrm{m}) \pm$ & \multicolumn{2}{|c|}{0.69} & \multicolumn{3}{|c|}{0.745} & \multicolumn{3}{|c|}{1.826} \\
\hline CD 5\% & \multicolumn{2}{|c|}{1.91} & \multicolumn{3}{|c|}{2.066} & \multicolumn{3}{|c|}{5.061} \\
\hline
\end{tabular}


Table.6 Effect of pruning time and genotypes on length of fruit $(\mathrm{cm})$

\begin{tabular}{|c|c|c|c|c|c|c|c|c|}
\hline \multirow{2}{*}{$\begin{array}{l}\text { Treats. } \\
\text { Pruning Time }\end{array}$} & \multicolumn{8}{|c|}{ Guava genotypes } \\
\hline & S1 & $\mathbf{S 2}$ & S3 & S4 & S5 & S6 & S7 & Mean \\
\hline P1 & 6.65 & 7.15 & 7.20 & 7.35 & 7.15 & 7.05 & 7.15 & 7.10 \\
\hline $\mathbf{P 2}$ & 6.45 & 7.05 & 7.25 & 7.20 & 7.50 & 7.25 & 7.05 & 7.11 \\
\hline $\mathbf{P 3}$ & 6.95 & 7.05 & 7.15 & 7.10 & 7.15 & 7.25 & 7.05 & 7.10 \\
\hline $\mathbf{P 4}$ & 6.80 & 7.15 & 7.25 & 7.55 & 7.45 & 7.05 & 7.25 & 7.21 \\
\hline P5 & 6.65 & 7.05 & 7.25 & 7.25 & 7.05 & 7.15 & 7.10 & 7.07 \\
\hline P0(Control) & 6.35 & 6.95 & 6.85 & 7.25 & 7.05 & 6.95 & 7.15 & 6.94 \\
\hline Mean & 6.64 & 7.07 & 7.16 & 7.28 & 7.23 & 7.12 & 7.13 & 7.09 \\
\hline \multirow[t]{2}{*}{ Year $2013 \& 2014$} & \multicolumn{2}{|c|}{ Pruning Time } & \multicolumn{3}{|c|}{ Guava genotypes } & \multicolumn{3}{|c|}{ Interaction $(\mathrm{P} \times \mathrm{S})$} \\
\hline & \multicolumn{2}{|c|}{ Pooled } & \multicolumn{3}{|c|}{ Pooled } & \multicolumn{3}{|c|}{ Pooled } \\
\hline $\mathrm{SE}(\mathrm{m}) \pm$ & \multicolumn{2}{|c|}{0.02} & \multicolumn{3}{|c|}{0.022} & \multicolumn{3}{|c|}{0.054} \\
\hline CD 5\% & \multicolumn{2}{|c|}{0.05} & \multicolumn{3}{|c|}{0.06} & \multicolumn{3}{|c|}{0.15} \\
\hline
\end{tabular}

Table.7 Effect of pruning time and genotypes on diameter of fruit $(\mathrm{cm})$

\begin{tabular}{|c|c|c|c|c|c|c|c|c|}
\hline \multirow{2}{*}{$\begin{array}{l}\text { Treats. } \\
\text { Pruning Time }\end{array}$} & \multicolumn{8}{|c|}{ Guava genotypes } \\
\hline & S1 & S2 & S3 & S4 & S5 & S6 & S7 & Mean \\
\hline P1 & 6.45 & 7.10 & 7.30 & 7.45 & 7.30 & 7.10 & 7.20 & 7.13 \\
\hline $\mathbf{P 2}$ & 6.35 & 7.10 & 7.30 & 7.20 & 7.50 & 7.30 & 7.10 & 7.12 \\
\hline P3 & 6.40 & 7.10 & 7.20 & 7.10 & 7.30 & 7.30 & 7.20 & 7.09 \\
\hline $\mathbf{P 4}$ & 6.30 & 7.20 & 7.40 & 7.60 & 7.70 & 7.20 & 7.30 & 7.24 \\
\hline P5 & 6.20 & 7.20 & 7.30 & 7.40 & 7.10 & 7.20 & 7.20 & 7.09 \\
\hline Po(Control) & 6.20 & 7.00 & 7.10 & 7.10 & 7.10 & 7.00 & 7.20 & 6.96 \\
\hline Mean & 6.32 & 7.12 & 7.27 & 7.31 & 7.33 & 7.18 & 7.20 & 7.10 \\
\hline \multirow[t]{2}{*}{ Year 2013 \& 2014} & \multicolumn{2}{|c|}{ Pruning Time } & \multicolumn{3}{|c|}{ Guava genotypes } & \multicolumn{3}{|c|}{ Interaction $(\mathrm{P} \times \mathrm{S})$} \\
\hline & \multicolumn{2}{|c|}{ Pooled } & \multicolumn{3}{|c|}{ Pooled } & \multicolumn{3}{|c|}{ Pooled } \\
\hline $\mathrm{SE}(\mathrm{m}) \pm$ & \multicolumn{2}{|c|}{0.02} & \multicolumn{3}{|c|}{0.02} & \multicolumn{3}{|c|}{0.055} \\
\hline CD 5\% & \multicolumn{2}{|c|}{0.05} & \multicolumn{3}{|c|}{0.06} & \multicolumn{3}{|c|}{0.15} \\
\hline
\end{tabular}

Table.8 Effect of pruning time and genotypes on average number of seeds per fruit

\begin{tabular}{|c|c|c|c|c|c|c|c|c|}
\hline \multirow{2}{*}{$\begin{array}{l}\text { Treats. } \\
\text { Pruning Time }\end{array}$} & \multicolumn{8}{|c|}{ Guava genotypes } \\
\hline & S1 & S2 & S3 & S4 & S5 & S6 & S7 & Mean \\
\hline P1 & 332.0 & 255.00 & 241.0 & 259.0 & 287.00 & 279.0 & 249.0 & 271.71 \\
\hline $\mathbf{P 2}$ & 313.0 & 241.00 & 260.0 & 280.0 & 291.00 & 257.0 & 278.0 & 274.29 \\
\hline $\mathbf{P 3}$ & 345.0 & 239.00 & 245.0 & 256.0 & 268.00 & 257.0 & 268.00 & 268.00 \\
\hline $\mathbf{P 4}$ & 346.0 & 257.00 & 289.0 & 290.0 & 243.00 & 234.0 & 273.0 & 276.00 \\
\hline P5 & 312.0 & 256.00 & 255.0 & 253.0 & 281.00 & 275.0 & 245.0 & 268.29 \\
\hline P0(Control) & 329.0 & 243.00 & 244.0 & 253.00 & 278.00 & 254.0 & 281.5 & 268.93 \\
\hline Mean & 329.5 & 248.5 & 255. & 265.1 & 274.6 & 259.3 & 265.7 & 271.2 \\
\hline \multirow[t]{2}{*}{ Year 2013 \& 2014} & \multicolumn{2}{|c|}{ Pruning Time } & \multicolumn{3}{|c|}{ Guava genotypes } & \multicolumn{3}{|c|}{ Interaction $(\mathrm{P} \times \mathrm{S})$} \\
\hline & \multicolumn{2}{|c|}{ Pooled } & \multicolumn{3}{|c|}{ Pooled } & \multicolumn{3}{|c|}{ Pooled } \\
\hline $\mathrm{SE}(\mathrm{m}) \pm$ & \multicolumn{2}{|c|}{0.333} & \multicolumn{3}{|c|}{0.360} & \multicolumn{3}{|c|}{0.882} \\
\hline CD 5\% & \multicolumn{2}{|c|}{0.924} & \multicolumn{3}{|c|}{0.998} & \multicolumn{3}{|c|}{2.445} \\
\hline
\end{tabular}


Table.9 Effect of pruning time and genotypes on shelf life of fruit (days)

\begin{tabular}{|l|c|c|c|c|c|c|c|c|}
\hline Treats. & \multicolumn{9}{|c|}{ Guava genotypes } \\
\hline Pruning Time & S1 & S2 & S3 & S4 & S5 & S6 & S7 & Mean \\
\hline P1 & 3.35 & 8.40 & 8.30 & 9.15 & 8.45 & 8.40 & 8.30 & 7.76 \\
\hline P2 & 3.45 & 8.25 & 8.30 & 9.40 & 8.20 & 8.05 & 8.15 & 7.69 \\
\hline P3 & 3.20 & 8.15 & 8.10 & 9.25 & 8.25 & 8.05 & 8.30 & 7.61 \\
\hline P4 & 3.30 & 8.15 & 8.25 & 9.10 & 8.15 & 8.10 & 8.15 & 7.60 \\
\hline P5 & 3.10 & 8.15 & 8.15 & 9.10 & 8.15 & 8.35 & 8.20 & 7.60 \\
\hline P0(Control) & 3.45 & 8.10 & 8.20 & 9.15 & 8.20 & 8.30 & 8.25 & 7.66 \\
\hline Mean & 3.31 & 8.20 & 8.22 & 9.19 & 8.23 & 8.21 & 8.23 & 7.65 \\
\hline Year 2013 \& 2014 & Pruning Time & Guava genotypes & Interaction (P×S) \\
\hline SE (m) $\mathbf{E}$ & Pooled & \multicolumn{3}{c|}{ Pooled } & \multicolumn{3}{c|}{ Pooled } \\
\hline CD 5\% & 0.054 & \multicolumn{3}{c|}{0.058} & \multicolumn{3}{c|}{ NS } \\
\hline
\end{tabular}

Table 10 Effect of Genotypes on pulp texture

\begin{tabular}{|c|c|c|}
\hline Treatment & \multicolumn{2}{|c|}{ Pulp texture } \\
\hline Guava genotypes & Mature & Ripe \\
\hline S1 & Soft & Mashy \\
\hline S2 & Very crisp & Very crisp \\
\hline S3 & Very crisp & Very crisp \\
\hline S4 & Very crisp & Very crisp \\
\hline S5 & Very crisp & Very crisp \\
\hline S6 & Very crisp & Very crisp \\
\hline S7 & Very crisp & Very crisp \\
\hline
\end{tabular}

Table.11 Effect of pruning time and genotypes on fruit fly infestation (\%)

\begin{tabular}{|c|c|c|c|c|c|c|c|c|}
\hline Treats. & Guava & enotypes & & & & & & \\
\hline Pruning Time & S1 & S2 & S3 & S4 & S5 & S6 & S7 & Mean \\
\hline P1 & 47.10 & 48.60 & 50.62 & 47.34 & 50.62 & 47.73 & 49.10 & 48.73 \\
\hline P2 & 40.70 & 40.17 & 39.45 & 37.94 & 40.00 & 40.84 & 39.50 & 39.80 \\
\hline P3 & 14.00 & 16.50 & 14.00 & 13.50 & 15.50 & 16.50 & 17.50 & 15.36 \\
\hline P4 & 11.50 & 13.50 & 13.50 & 12.00 & 13.00 & 14.00 & 15.00 & 13.21 \\
\hline P5 & 7.50 & 10.50 & 10.00 & 8.50 & 10.50 & 10.50 & 12.50 & 10.00 \\
\hline P0(Control) & 46.99 & 48.00 & 49.00 & 46.00 & 46.50 & 47.89 & 47.99 & 47.48 \\
\hline Mean & 27.97 & 29.55 & 29.43 & 27.55 & 29.35 & 29.58 & 30.27 & 29.10 \\
\hline Year 2013 \& 2014 & Pruni & Time & & va geno & ypes & & raction & $x S)$ \\
\hline & & & & Pooled & & & Pooled & \\
\hline $\mathrm{SE}(\mathrm{m}) \pm$ & & & & 0.903 & & & 2.211 & \\
\hline CD 5\% & & & & NS & & & NS & \\
\hline
\end{tabular}


Table.12 Effect of pruning time and genotypes on marketable Yield per plant free from fruit fly infestation $(\mathrm{kg})$

\begin{tabular}{|c|c|c|c|c|c|c|c|c|}
\hline Treats. & & & & uava $g$ & otypes & & & \\
\hline Pruning Time & S1 & S2 & S3 & S4 & S5 & S6 & S7 & Mean \\
\hline P1 & 17.68 & 14.18 & 16.64 & 16.43 & 13.80 & 16.52 & 12.74 & 15.42 \\
\hline P2 & 17.59 & 17.25 & 19.70 & 20.92 & 15.85 & 18.51 & 17.72 & 18.22 \\
\hline P3 & 31.84 & 27.52 & 26.91 & 31.82 & 25.69 & 27.99 & 25.85 & 28.23 \\
\hline $\mathbf{P 4}$ & 21.55 & 20.53 & 20.53 & 19.57 & 20.76 & 18.92 & 18.40 & 20.04 \\
\hline P5 & 15.69 & 17.53 & 12.90 & 13.57 & 12.82 & 13.86 & 12.85 & 14.17 \\
\hline P0(Control) & 16.89 & 13.89 & 15.70 & 17.37 & 13.91 & 15.60 & 13.68 & 15.29 \\
\hline Mean & 20.21 & 18.48 & 18.73 & 19.95 & 17.14 & 18.56 & 16.87 & 18.56 \\
\hline Year $2013 \& 2014$ & Pruni & Time & & Ja geno & & & raction & \\
\hline & & & & Pooled & & & Poolec & \\
\hline $\mathrm{SE}(\mathrm{m}) \pm$ & & & & 0.214 & & & 0.525 & \\
\hline CD 5\% & & & & 0.594 & & & 1.455 & \\
\hline
\end{tabular}

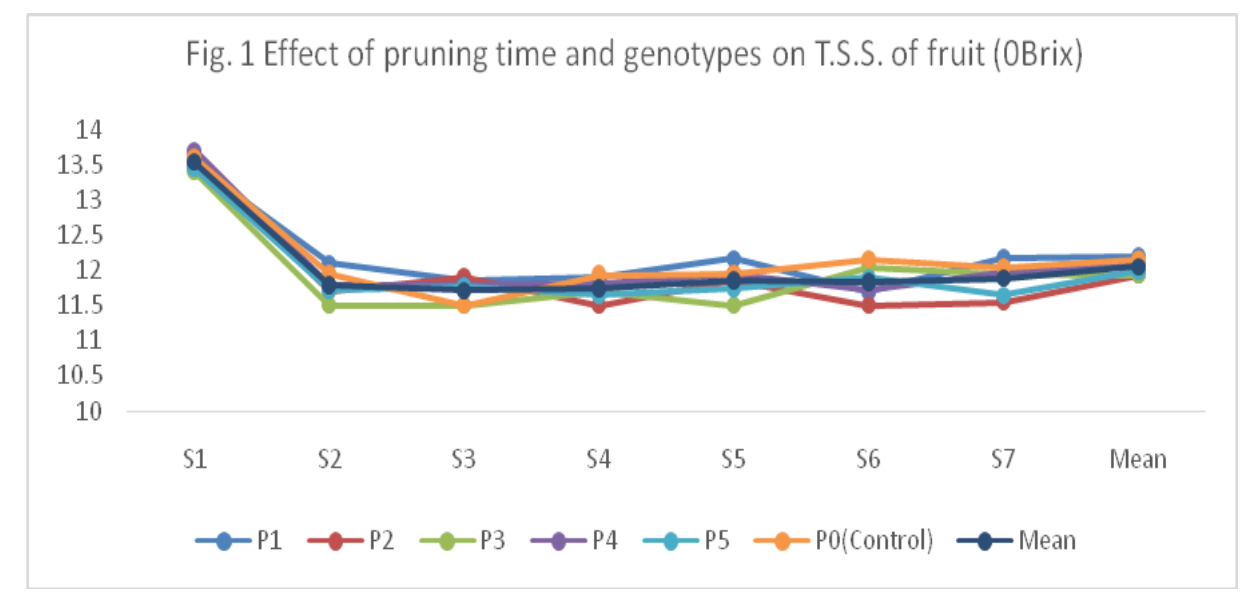

Fig. 2 Effect of pruning time and genotypes on total sugars of fruit (\%)

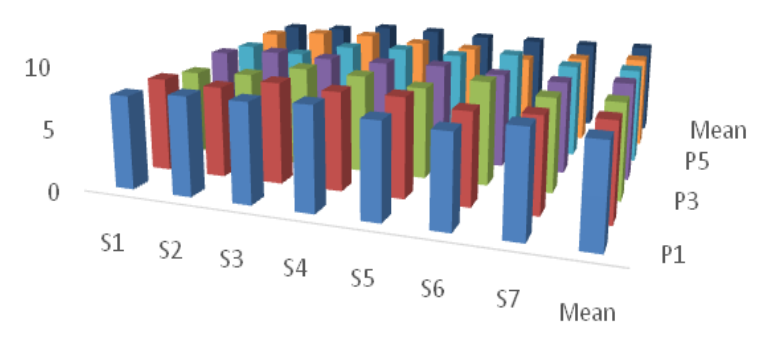

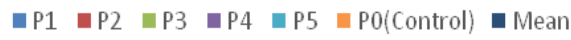



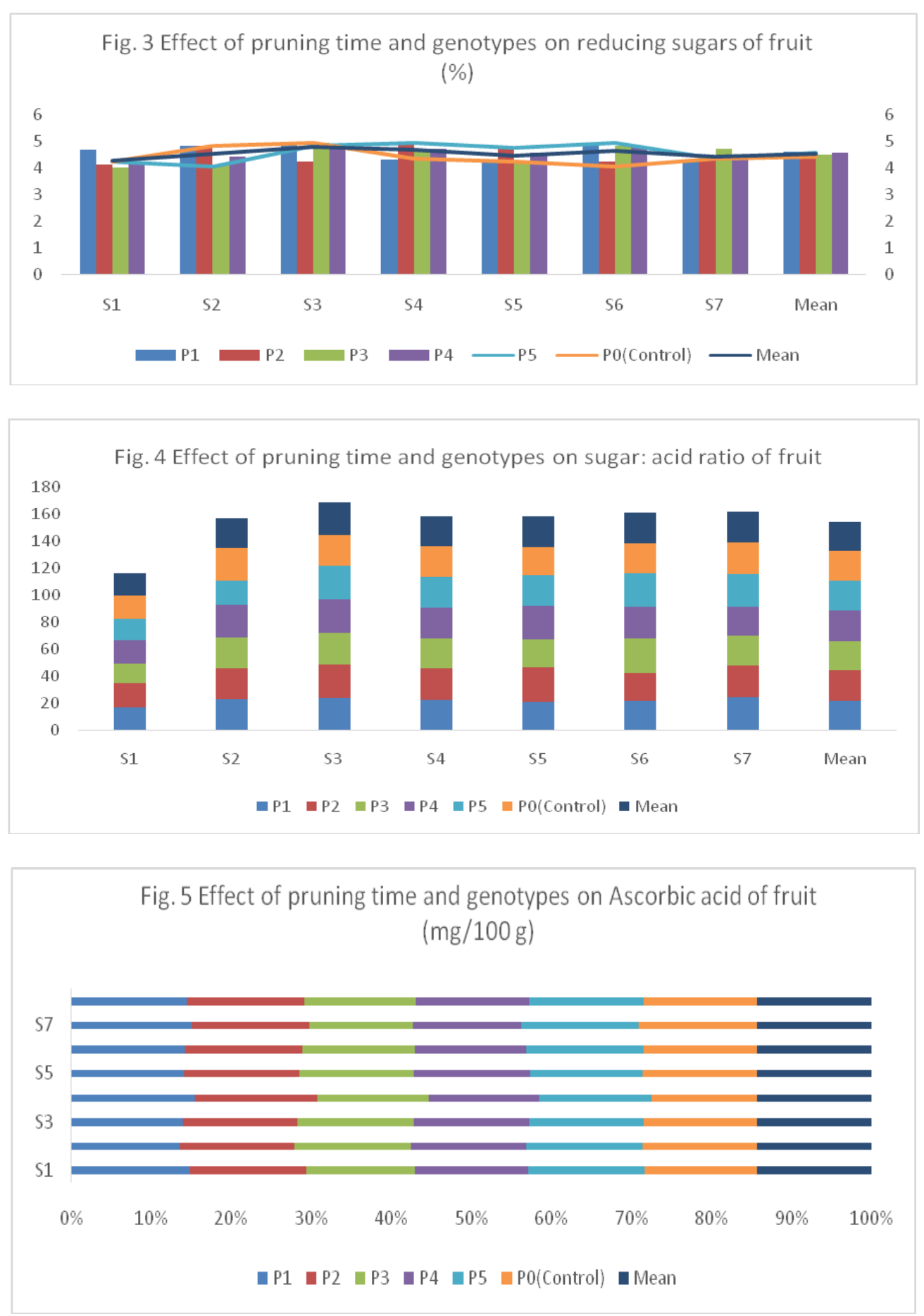

TSS

As regards, the effect of genotypes was found to be significant, maximum $\left(13.54^{0} \mathrm{Brix}\right) \mathrm{TSS}$ was noted in $\mathrm{S}_{1}$ (Sardar) Cv. (Fig. 1). The result of the conducted experiment shows that time of pruning does not affect TSS too much.
But, different genotypes get affected by pruning in that maximum TSS recorded in $\mathrm{S}_{1}$ treatment and less TSS was recorded in control ones. This is due to the effect of pruning of plants, attributed to lower leaves/ fruit ratio in such trees. Pruning time was not affecting too much acid. Similar results 
regarding the effect of pruning on TSS of fruits were recorded by Bajpai et al., (1973) and Sheikh and Hulmani (1996). They also reported an increased fruit TSS with pruning in guava.

\section{Total sugars and reducing sugars}

Effect of pruning time and genotypes were found to be non-significant, but more total and reducing sugars were found in $\mathrm{S}_{3}$ (RHRGuv-60) (Fig. 2 and 3). The improvement was observed in quality of guava fruit of pruned trees compared to control one were better. Bajpai et al., (1973), Sing et al., (2005) reported that better quality of fruits observed in fruits of pruned guava plants compared to control ones.

\section{Acidity}

This is very important biochemical parameter decides taste blend of guava. Non-significant differences due to different pruning time were observed. Data of effect of genotypes were observed to be significant. However, Maximum (0.43\%) acidity was noted in S1 (Sardar) (Fig. 4). Pruning time was not affecting too much acidity. But the genotypes differed in acidity; it might due to the independent characteristic of genotype along with pruning effect and also might be due to the abundant availability of photosynthesis for a limited number of fruits leads to increase in acidity.

\section{Sugar: acidity ratio}

With respect to genotypes, significantly maximum sugar: acid ratio (24.1) was noticed in $\mathrm{S}_{3}$ (RHR-Guv-60) and effect of time of pruning and interaction between different times of pruning and genotypes was found to be non-significant for sugar: acid ratio (Fig. 4). In obtained results, maximum sugar acid ratio was noticed in pruned trees as compared to control trees of Guava. This is might be due to healthy shoot canopy, better sunlight distribution in the canopy, better sunlight utilization and better photosynthetic rate in pruned plants. Shirsath (2013) reported that maximum sugar acid ratio was recorded in pruned plants compared to control ones.

\section{Ascorbic acid}

Pooled results revealed that significantly maximum (199.93 mg/100 g) ascorbic acid was observed in $\mathrm{P}_{2}\left(15^{\text {th }}\right.$ June). In case of the data of effect genotypes, maximum (202.17 $\mathrm{mg} / 100 \mathrm{~g}$ ) ascorbic acid was observed in $\mathrm{S}_{4}$ (RHR-Guv-14).In the obtained results, ascorbic acid content in fruit increased with pruning as compared to control ones (Fig. 5). This might be due to the abundant availability of photosynthesis for a limited number of fruits leads to increase in ascorbic acid. As well as the prevalence of low temperature increases ascorbic acid in the fruit. Sheikh and Hulmani (1996) and Kaur (1999), who registered the highest ascorbic acid content in fruits produced by trees subjected to severe pruning, also observed improved ascorbic acid content in fruits of guava after pruning.

\section{The shelf life of fruit}

As regards data on genotypes, the maximum shelf life of fruit (9.19 days) was noticed in $\mathrm{S}_{4}$ (RHR-Guv-14) and effect of time of pruning and interaction between different times of pruning and genotypes was found to be nonsignificant (Table 9). The maximum shelf life of fruit, it might be due to a low rate of respiration of fruit due to which slow degradation of fruit taking place.

\section{Pulp texture}

Fruit of genotypes $\mathrm{S}_{2}, \mathrm{~S}_{3}, \mathrm{~S}_{4}, \mathrm{~S}_{5}, \mathrm{~S}_{6}$ and $\mathrm{S}_{7}$ having very crisp pulp texture at a mature stage and also at ripe stage (Table 10). 
Genotype $S_{1}$ has soft pulp texture at the mature stage of fruit and mashy pulp texture at the ripe stage of fruit. Pulp texture is the very important quality parameter of fruits of guava that is related to less or more preferably of fruits of guava by consumers in the market.

\section{Fruit fly infestation}

Significantly lowest fruit fly infestation (10 $\%)$ was recorded in $\mathrm{P}_{5}\left(15^{\text {th }}\right.$ Sept.) followed by $\mathrm{P}_{4}\left(15^{\text {th }}\right.$ Aug.) $(13.25 \%)$ time of pruning. Effect of genotypes and interaction between different times of pruning and genotypes was found to be non-significant for fruit fly infestation. This is due to the change in the time of fruiting and harvesting by pruning operation. Fruit fly infestations were recorded maximum in rainy season compared to winter season crop (Table 11). When the pruning is done in Aug-Sept, the fruit will be available in Feb-March meanwhile incidence of fruit flies in too much less. The finding of present studies are found in consonance with that Shirsath (2013) reported that less incidence of fruit fly was recorded in pruned guava plants as compared to control ones. Similarly, Muhammad et al., (2014) reported that the abundance of fruit fly was observed throughout the year, with two peaks in summer from May to August and during winter from November to January coinciding with availability of guava fruits. The maximum fruit damage (18.59\%) occurred in August, and the second peak with $13.37 \%$ damage observed during the period of July.

\section{Total yield per plant free from infestation}

With respect to pruning time, yield per plant (kg) free from infestation was recorded significantly maximum $(28.23 \mathrm{~kg})$ in P3 (15th July). Highest $(20.21 \mathrm{~kg})$ yield per plant was noted in S1 (Sardar) (Table 12). Findings of the present studies in line with those Anon., (1979) and Rao and Khader (1980), who obtained the higher mean yields over seven years with pruning as compared to no pruning in mango. Likewise, Shirsath (2013) reported that there is an increase in the yield of moderate pruned trees as compared to compared to unpruned guava trees. Bajpaiet al., (1973) and Benington (1981) reported that the fruit yield increased significantly with light pruning in guava and Valencia orange trees, respectively. On the contrary, Jadhavet al., (1998) obtained the highest yield in guava with severe pruning, i.e., pruning $60 \mathrm{~cm}$ from the tip.

In conclusion the genotype RHR-Guv-14 was found to be better in quality like lustrous fruit, crispy pulp texture, large fruit size, more fruit weight, maximum shelf life of fruit, that's why it can be evaluate for cultivation as hasta bahar crop. Similarly, 15 Sept pruning timewas found to be important in escape from fruit fly infestation and with respect to marketable yield 15th July pruning time was found to be better.

\section{References}

Anonymous, 1979. Mangoes pruning methods evaluated. Information Bull, Citrus, and Subtropical Fruit Research Institute, South Africa 80: 8-9 (Horticultural Abstracts 51; Entry No. 837).

Anonymous, 2014. National Horticulture Database, 2013. NHB pp: 76-82. http://www.nhb.gov.in

Aravindakshan, M. 1963. Effect of pruning on growth, flowering and furit set of guava (Psidium guajava L.) Madras Agric. J., 51: 87-90.

Bajpai, P.N., Shukla, H.S. and Chaturvedi A.M. 1973. Effect of pruning on growth, yield, and quality of guava (Psidiumguajava L.) var. Allahabad Safeda. Prog. Hort., 5: 73-79.

Basu, J., Das, B., Sarkar, S., Mandal, K.K., Banik, B.C., Kundu, S., Hasan, M.A., Jha, S. and Ray, S.K. 2007. Studies on response of pruning for rejuvenation of old guava orchard. Acta. Hort.735: 303-309.

Bevington, K.B. 1981. The response of Valencia 
orange trees to hedging and topping. Proc. Flor. Sta. Hort. Soc.,93: 65-66 (Horticultural Abstracts 51: Entry No. 9754).

Dasarathi, T.B. 1951. The guava. Madras Agric. J. 38: 520-26.

Dhaliwal, H.S., Banke, A.K., Sharma, L.K. and Bali, S.K. 2014. Impact of pruning practices on shoot growth and bud production in Kinnow (Citrus reticulate Blanko) plant. $J$. Exper. Bio. andAgril. Sci., 1(7): 507-513.

Fivaz, J. and Stassen, P.J.C. 1994. The effect of time of pruning and fruit thinning on yield and fruit quality of 'Sensation' mangoes. Yearbank South African Mango Growers Association, 14:49-54.

Gill, H.S. 1994. Pruning studies on Sardar guava. M.Sc. Thesis, Punjab Agric. Univ., Ludhiana, India.

Haseeb, M. 2007. Current status of insect pest production in guava. Acta Horti., (ISHS) 735:453-467.

Jadhav, B.J., Damke, M.M. Mahorkar, V.K., Dod, V.N. and Wagh, A.P. 1998. Studies on the effect of time and severity of pruning on growth and yield of guava (Psidium guajava L.) cv. Sardar. J. Soils and Crops, 8: 2,13941; 6 refs 51 (CAB Abstracts 1998: AN: 990301545).

Kaur R. 1999. Crop regulation by pruning in Sardar guava. M.Sc. Thesis, Punjab Agric. Univ, Ludhiana India.

Lal, B., Rajput, M.S. and Rathore, D.S. 2000. Effect of pruning on renovation of old mango trees. Indians J. Hort., 57(3):240-242.

Lotter, J. De. V. and Lotter, De. V.J. 1990. Vegetative and reproductive habit of guava in relation to pruning methods. Acta Horticulture, 275: 229-37.

Mahor, M.K., Tiwari, R., and Baghel, B.S. 2012. Physico-chemical characteristics of different varieties of guava in Malva Plateau of Madhya Pradesh. Agric.Sci. Digest, 32(2): 141:144.

Muhammad, S., Muhammad, H, Muhammad, Y. and Mureed, H. 2014. Monitoring of Population Dynamics and Fruits Infestation of Tephritid Fruit Flies (Diptera: Tephritidae) in Guava (Psidium guajava L.) Orchard. Res. and Rev.: J. of Agri. and Alli. Sci., 3(2): 3640.

Rao, V.N.M., and Khader, J.B. 1980. Effect of pruning and thinning of young shoot clusters of mango. Sci. and Cult., 46: 71-72 (Horticulture Abstracts 51: AN: 4410).

Sheikh, M.K. and Hulmani, N.C. 1996. Effect of severity of pruning on flowering and fruit quality of guava cv. Navalur. Prog Hort., 25: 157-60.

Shirsath, H.K. 2013. Studies on agro-techniques in guava (PsidiumguajavaL.) cv. SardarI. High-density planting and II. Rejuvenation of old orchard. Ph.D. Thesis, Mahatma Phule Krishi Vidyapeeth, Rahuri, Ahmednagar, India.

Singh, G., Rajan, S. and Singh, A.K. 2001. Influence of pruning date on fruit yield of guava under subtropics. J. Appl. Horti., 3(1): 37-40.

Singh, G., Mishra, Rajneesh and Singh, G.P. 2005. Guava Rejuvenation, Extension Bulletin-28. CISH, Lucknow, pp-20.

Sundarajan, S. and Muthuswamy, S. 1964a. Effect of pruning on fruit size and weight in certain varieties of guava (Psidium guajava L.) South Ind. Hort., 17: 21-24.

Tiwari, J.P. and Lal, S. 2007. Effect of NAA, flower bud thinning and pruning on crop regulation in Guava (Psidium guajava L.) Cv. Sardar. Proc. Ist IS on Guava. Acta Hort. 735, ISHS, 2007.

\section{How to cite this article:}

Nikumbhe, P. H., J. Uchoi and Mali, D. S. 2020. Effects on Tree Vigor, Fruit Quality, Yield and Fruit Fly Infestation of Different Time of Pruning with Genotypes in Guava (Psidium guajava L.). Int.J.Curr.Microbiol.App.Sci. 9(11): 1769-1780.

doi: https://doi.org/10.20546/ijcmas.2020.911.209 